\title{
Quantum Dots in a Strong Magnetic Field. Quasi-classical consideration
}

\author{
A. Matulis \\ Institute of Semiconductor Physics, Goštauto 11, 2600 Vilnius, Lithuania
}

(Dated: November 2, 2018)

\begin{abstract}
The electron motion in rather strong magnetic fields (when only the lowest Landau level is populated) is considered. In this case the electron kinetic energy is frozen out and the electrons are guided by slowly varied potential. Using the adiabatic procedure and expansion in magnetic length series the approximate description is developed. In zero order this approximation leads to the classical equations of motion describing the Larmor circle drift in the potential gradient. In the second order the special quantum mechanical description where the electron potential energy plays the role of the total Hamiltonian is constructed. Simple examples of a single and two electrons in the parabolic dot demonstrates that the proposed approximate description gives the main features of the electron system spectrum and the collective phenomena.
\end{abstract}

\section{INTRODUCTION}

Quantum dots, or artificial atoms, have been a subject of intense theoretical and experimental research over the last few years [1]. The useful instrument in spectroscopy experiments is the magnetic field applied in perpendicular to the quantum dot plane direction which enables to trace easily the dependence of the quantum dot properties on various parameters. Moreover the strong magnetic field reveals the quantization effects introducing into the electron system the favorable interplay between confining potential and Landau levels.

Recently the main interest in quantum dots is related to the electron-electron interaction and the collective phenomena, such as the change of the ground state multiplicity, the electron density reconstruction, and the Wigner crystallization. The electron density reconstruction in the finite electron systems was considered in [2]. Now it is known as Shamon-Wen edge - some of the electron density ring around the finite system. Under certain circumstances the ring was reported to become unstable [3], and it breaks into separate lumps. Although the possibility to obtain the symmetry braking solutions was argued [4] considering them as an artifacts of the approximate methods used, the exact calculations of the electron correlation function [5] undoubtedly indicates that the Wigner crystallization occurs at rather large electronelectron interaction. The presented in [6] electron density plots show that the strong magnetic field facilitates the electron density edge reconstruction leading to the Wigner crystallization.

Meanwhile the minimization of the system potential presented in [7] shows that the Wigner crystallization in quantum dots can be successfully considered by classical mechanics. The fact that the strong magnetic field facilitates the Wigner crystallization enables to suppose that the electron system behavior in very strong magnetic can

\footnotetext{
*E-mail me at: amatulis@takas.lt
}

be described by classical or quasi-classical methods. The purpose of the present paper is to show how such methods could be developed. The paper is organized as follows. After the formulation of the model in the next Section, in Sections 3 and 4 the main instrument - fast and slow variables are introduced. Then in Section 5 the adiabatic procedure is discussed and the slow motion Schrödinger equation is considered. In Section 6 the classical equations for the limit case of strong magnetic field are derived, and in the next two Sections the illustrations of the simplified quantum mechanical description are given. In Appendix A the details of the adiabatic procedure are presented, and in Appendix B the transformation back to the initial coordinates is discussed.

\section{MODEL}

We consider the Schrödinger equation

$$
i \hbar \frac{\partial}{\partial t} \Psi=H_{T} \Psi
$$

with the Hamiltonian

$$
H_{T}=\frac{1}{2 m}\left\{\boldsymbol{p}+\frac{e}{c} \boldsymbol{A}(\boldsymbol{r})\right\}^{2}+V(\boldsymbol{r})
$$

describing the motion of $2 \mathrm{D}$ electrons in the strong perpendicular homogeneous magnetic field and slowly varying potential $V(\boldsymbol{r})$. For the sake of simplicity the main equations will be derived for a single electron as the generalization for the system of many electrons is trivial. It will be presented at the end of derivation.

Choosing the symmetric gauge $\boldsymbol{A}=[\boldsymbol{B} \times \boldsymbol{r}]$ we write down the main part of the Hamiltonian as follows:

$$
H_{0}=\frac{1}{2 m}\left\{\left(p_{x}-\frac{e B}{2 c} y\right)^{2}+\left(p_{y}+\frac{e B}{2 c} x\right)^{2}\right\}
$$

We shall consider it as a largest one treating the remaining potential $V(\boldsymbol{R})$ as a small perturbation. 


\section{LANDAU LEVELS}

As in the standard perturbation technique we have to start with the zero order problem and solve the following stationary Schrödinger equation:

$$
\left\{H_{0}-\varepsilon\right\} \psi=0 \text {. }
$$

The solution of it is known as Landau levels. The most simple way to obtain it is to introduce the new variables

$$
\xi=\frac{l_{B}}{\hbar} p_{x}-\frac{1}{2 l_{B}} y, \quad \eta=\frac{l_{B}}{\hbar} p_{y}+\frac{1}{2 l_{B}} x
$$

where $l_{B}=\sqrt{c \hbar / e B}$ is the magnetic length. Using the new variables Hamiltonian (3) can be rewritten as

$$
H_{0}=\frac{\hbar \omega_{c}}{2}\left(\xi^{2}+\eta^{2}\right)
$$

where $\omega_{c}=e B / m c$ is the cyclotron frequency, and the new variables obey the following commutation rule:

$$
[\xi, \eta]=-i
$$

The zero order Hamiltonian reminds the Hamiltonian of the harmonic oscillator, and it is evident that it has the equidistant discrete spectrum which as it was already mentioned is called the Landau levels.

We shall consider the case of very strong magnetic field when the electrons are in the lowest Landau level. Our task is to reveal how the slowly varying additional potential $V(\boldsymbol{r})$ (as compared with the magnetic length $l_{B}$ ) changes their behavior.

\section{SLOW VARIABLES}

We shall treat the variables introduced in the previous Section as fast variables because they are included into the main part of the Hamiltonian. But as we are going to solve the $2 \mathrm{D}$ problem they are not sufficient to treat initial Schrödinger equation (1). We have to introduce two more variables. We shall do that in the following way:

$$
X=\frac{1}{2} x-\frac{l_{B}^{2}}{\hbar} p_{y}, \quad Y=\frac{1}{2} y+\frac{l_{B}^{2}}{\hbar} p_{x} .
$$

We chose them in such way in order to have the most simple commutation relations, namely,

$$
[\xi, X]=[\xi, Y]=[\eta, X]=[\eta, Y]=0,
$$

and

$$
[Y, X]=-i l_{B}^{2}
$$

We shall consider those variables as slow ones.

Now substituting the initial variables

$$
x=X+l_{B} \eta, \quad y=Y-l_{B} \xi
$$

into Hamiltonian (2) we arrive at the following expression:

$$
H_{T}=\frac{\hbar \omega_{c}}{2}\left(\xi^{2}+\eta^{2}\right)+V\left(X+l_{B} \eta, Y-l_{B} \xi\right) .
$$

So, we divided the Hamiltonian into two parts. The first largest one describing the motion of the electron in the homogeneous magnetic field depends on the fast variables only, while the other one - the slowly varying potential - depends on both fast and slow variables. Thus, we see that the slow and fast variables can not be separated exactly, but the presence of the small parameter (namely, the ratio of the magnetic length $l_{B}$ and the characteristic potential variation length $\left.l_{0} \sim|V / \nabla V|\right)$ enables us to separate them approximately by means of some adiabatic procedure.

\section{ADIABATIC PROCEDURE}

Now we are going to develop some adiabatic procedure and apply it for considering the Schrödinger equation (11). For this purpose we shall make the following steps:

- we expand the potential into $l_{B}$-powers:

$$
\begin{aligned}
V & =V(X, Y)+l_{B} \eta V_{X}(X, Y) \\
& -l_{B} \xi V_{Y}(X, Y)+\cdots ;
\end{aligned}
$$

- divide the Hamiltonian into two parts:

$$
\begin{aligned}
H & =H_{f}+H_{s} \\
H_{f} & =\frac{\hbar \omega_{c}}{2}\left(\xi^{2}+\eta^{2}\right) \\
& +l_{B} \eta V_{X}(X, Y)+l_{B} \xi V_{Y}(X, Y)+\cdots, \\
H_{s} & =V(X, Y)
\end{aligned}
$$

- present the wave function as the product of its fast and slow parts:

$$
\Psi=\psi(\eta \mid X, Y) \Phi(X)
$$

- and use the following equation for the fast wave function part:

$$
\left\{H_{f}-E(X, Y)\right\} \psi(\eta \mid X, Y)=0 .
$$

Actually it is the standard adiabatic procedure which has to lead to the Schrödinger equation for the slow electron motion

$$
i \hbar \frac{\partial}{\partial t} \Phi(X)=H \Phi(X),
$$

with the effective slow motion Hamiltonian

$$
H=V(X, Y)+E(X, Y) .
$$

However, there are some peculiarities caused by the fact that according to Eqs. (7, 10) neither fast nor slow 
variables commute each with other. That is why both wave function parts in Eq. (117) depend only on a single variable (either $\eta$ or $X$ ), while the other one has to be treated as an operator $\left(\xi=-i \partial / \partial \eta, Y=-l_{B}^{2} \partial / \partial X\right)$. Consequently, $X$ and $Y$ variables entering the fast wave function part $\psi(\eta \mid X, Y)$ and the corresponding eigenvalue $E(X, Y)$ can not be treated as parameters (what is done in the standard adiabatic procedure), but should be considered as the operators acting on the slow wave function part. This makes the adiabatic procedure a little bit tricky and cumbersome. Nevertheless due to the presence of the small parameter $l_{B} / l_{0}$ it can be performed. The details of this derivation are presented in the Appendix A. Restricting the consideration up to the $l_{B}^{2}$ order we shall use the following slow motion Hamiltonian:

$$
H=V^{(S)}(\boldsymbol{R})+\frac{l_{B}^{2}}{4} \nabla^{2} V^{(S)}(\boldsymbol{R}) .
$$

The superscript ${ }^{(S)}$ indicates that the expression should be symmetrized in respect of the permutation of the slow variables $X$ and $Y$ which as we know already do not commute each with other.

The above adiabatic procedure can be easily generalized for the case of many electron system. As the slow motion different electron coordinates $\boldsymbol{R}_{i}$ commute each with other this generalization reduces to inserting the proper summations into obtained slow motion Hamiltonian and replacing it by the following expression:

$$
H=V^{(S)}\left(\boldsymbol{R}_{1}, \boldsymbol{R}_{2}, \cdots\right)+\frac{l_{B}^{2}}{4} \sum_{i=1}^{N} \nabla_{i}^{2} V^{(S)}\left(\boldsymbol{R}_{1}, \boldsymbol{R}_{2}, \cdots\right) .
$$

Now we are going to consider some simple examples in order to illustrate the application of the proposed simplified description of the motion of electrons in the case of strong magnetic fields. Let us start with the zero order $\left(l_{B}=0\right)$ approximation.

\section{CLASSICAL EQUATIONS OF MOTION}

In zero order approximation we shall take into account only the first term in Hamiltonian (21) and neglect the commutator (10) between $X$ and $Y$ coordinates. We know that neglecting the commutators we have to arrive to the classical mechanics. But one has to remember that it is not correct just to neglect the commutators. It is necessary to replace them by the corresponding Poisson brackets according to the following rule (note we inserted $l_{B}^{2}$ instead of $\left.\hbar\right)$ :

$$
\frac{i}{l_{B}^{2}}[A, B] \quad \rightarrow \quad\{A, B\}=\frac{\partial A}{\partial y} \frac{\partial B}{\partial x}-\frac{\partial A}{\partial x} \frac{\partial B}{\partial y} .
$$

The most simple way to obtain the classical equations of motion is to use the Heisenberg equations of motion for the operators. Thus, we write

$$
\begin{aligned}
\frac{d}{d t} X & =\frac{i}{\hbar}[H, X]=\frac{l_{B}^{2}}{\hbar} \frac{i}{l_{B}^{2}}[H, X] \\
& \rightarrow \frac{l_{B}^{2}}{\hbar}\{H, X\}=\frac{c}{e B} \frac{\partial V}{\partial Y}, \\
\frac{d}{d t} Y & =-\frac{c}{e B} \frac{\partial V}{\partial X} .
\end{aligned}
$$

Note in the Heisenberg equations of motion the Plank constant $\hbar$ is used (in spite of the fact that commutator of the variables is proportional to the magnetic length squared), because it has to be in agreement with the slow motion Schrödinger equation (19). Those two equations of motion can be rewritten as a single vector equation

$$
\dot{\boldsymbol{R}}=-\frac{c}{e B}\left[\boldsymbol{e}_{z} \times \nabla\right] V(\boldsymbol{R})
$$

where the symbol $\boldsymbol{e}_{z}$ stands for the unit vector perpendicular to the electron motion plane $z=0$. It is well known equation in plasma physics, and it describes the Larmor circle (the rotating electron in a strong magnetic field) drift caused by the gradient of applied additional potential.

Thus, we see that system of $2 \mathrm{D}$ electrons in the very strong magnetic field (in the conditions of the fractal Hall effect, when only the part of the lowest Landau level is populated) demonstrates the classical behavior. This classical behavior is rather tricky. They do not behave as electrons. They behave as a system of classical gyroscopes.

Now let us go back and take the $l_{B}^{2}$ order terms into account. In this case the quantum mechanical correction should take place, and we have to obtain something like quasi-classical description. In order to understand the main features of such quasi-classical motion let us take the most simple example of the parabolic dot with one and two electrons.

\section{SINGLE ELECTRON IN A PARABOLIC DOT}

In order to check the correctness of the above described method let us start with trivial problem of a single electron in a parabolic dot. In this case we have the following potential:

$$
V(\boldsymbol{r})=\frac{m \omega_{0}^{2}}{2} r^{2}
$$

with the frequency $\omega_{0}$ characterizing the strength of the confining potential, and according to Eq. (21) the following slow motion Hamiltonian:

$$
\begin{aligned}
H & =\frac{m \omega_{0}^{2}}{2}\left(X^{2}+Y^{2}\right)+\frac{1}{2} m \omega_{0}^{2} l_{B}^{2} \\
& =\frac{m \omega_{0}^{2}}{2}\left\{-l_{B}^{4} \frac{\partial^{2}}{\partial X^{2}}+X^{2}+\frac{1}{2} l_{B}^{2}\right\} .
\end{aligned}
$$




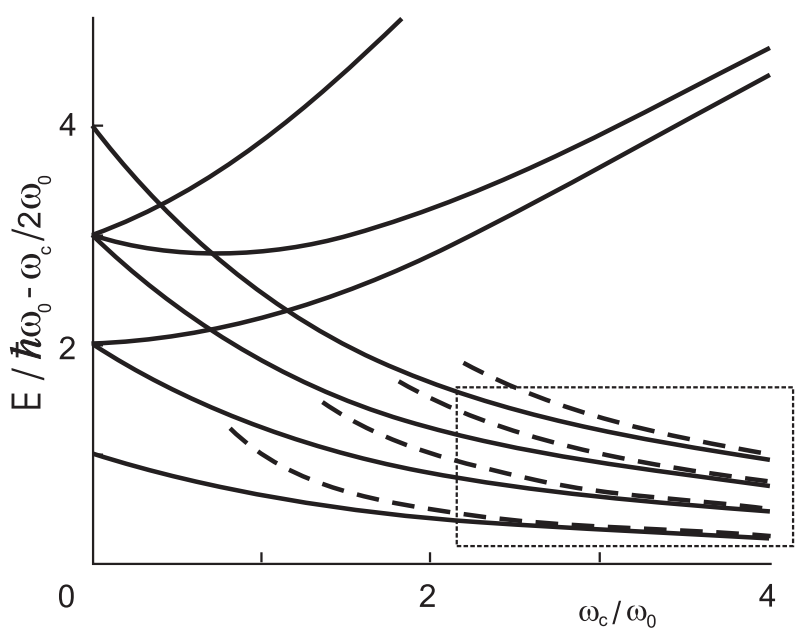

FIG. 1: Electron spectrum in a parabolic dot: solid curves - the exact result according (31), dashed curves — the slow motion approximation (29).

This the well known Hamiltonian of the harmonic oscillator. Its eigenvalues and the corresponding eigenfunctions are

$$
\begin{aligned}
E_{n} & =\frac{\hbar \omega_{0}}{\gamma}(n+1), \\
\Phi_{n}(X) & =\frac{1}{\sqrt{l_{B} 2^{n} n ! \sqrt{\pi}}} \mathrm{e}^{-X^{2} / 2 l_{B}^{2}} H_{n}\left(X / l_{B}\right)
\end{aligned}
$$

where the parameter $\gamma=\omega_{c} / \omega_{0}$ characterizes the relative strength of the magnetic field, and the symbol $H_{n}$ stands for the Hermit polynomial.

In order to evaluate the approximation obtained by solving the slow motion Schrödinger equation let us compare it with the exact Fock-Darvin result which is

$$
E_{n m}=\hbar \omega_{0}\left\{(2 n+|m|+1) \sqrt{1+\gamma^{2} / 4}+(m-1) \gamma / 2\right\}
$$

where orbital quantum number $m$ is integer, and radial quantum number $n$ is integer and nonnegative. This exact result together with the approximate one (29) are shown in Fig. 11 by solid and dashed curves, correspondingly. We see that in the asymptotic region $\gamma \rightarrow \infty$ (shown by the dotted rectangular) the approximate result is rather close to the rotational levels belonging to the lowest Landau level. Moreover, we may expect the quantitative agreement already at $\gamma \gtrsim 2$ values. It is interesting to inspect how the wave function and the corresponding electron density looks like. However, we have to remember that eigenfunction (30) is not the electron wave function itself but it is its slow motion part only. In order to obtain the electron wave function according to Eq. (17) we have to multiply it by fast motion part. Next we have to go back to the initial variables (11). It can be done using some integral transformation which is described in Appendix B. Using transformation kernel (B6) and restricting our consideration by the lowest fast wave function approximation (A16) we write down the total electron wave function in initial $x, y$ variables

$$
\begin{aligned}
\Psi_{n}(x, y)=\int_{-\infty}^{\infty} d \eta \int_{-\infty}^{\infty} d X\langle x, y \mid \eta, X\rangle \psi_{0}(\eta) \Phi_{n}(X) \\
=\frac{1}{2 \pi l_{B} \sqrt{2^{n} n !}} \int_{-\infty}^{\infty} d \eta \int_{-\infty}^{\infty} d X H_{n}\left(X / l_{B}\right) \\
\cdot \mathrm{e}^{-\eta^{2} / 2+i y\left(X-l_{B} \eta\right) / 2 l_{B}^{2}-X^{2} / 2 l_{B}^{2}} \delta\left(X+l_{B} \eta-x\right) \\
=\frac{\mathrm{e}^{\left(i x y-x^{2}\right) / 2 l_{b}^{2}}}{2 \pi l_{B} \sqrt{2^{n} n !}} \int_{-\infty}^{\infty} d \eta \mathrm{e}^{-\eta^{2}+(x-i y) \eta / l_{B}} H_{n}\left(x / l_{B}-\eta\right) .
\end{aligned}
$$

Fortunately the integral can be calculated by analytical means. Using the standard integrals with Hermit polynomials [8] we obtain the following expression for the total electron wave function

$$
\Psi_{n}(x, y)=\frac{1}{l_{B} \sqrt{2^{n+1} n ! \pi}} \mathrm{e}^{i n \varphi}\left(r / l_{B}\right)^{n} \mathrm{e}^{r^{2} / 4 l_{B}^{2}},
$$

and the corresponding electron density in the $n$ eigenstate

$$
\rho_{n}(\boldsymbol{r}) \sim\left(r / l_{B}\right)^{2 n} \mathrm{e}^{-r^{2} / 2 l_{B}^{2}} .
$$

We see that in the case of large $n$ values (in the quasiclassical case) the electrons are mainly located on the ring. Equating to zero the derivative of the above density expression we obtain the radius of this ring. It is

$$
r_{0}=l_{B} \sqrt{2 n} .
$$

Now inserting the $n$ value expressed from Eq. (29) we get

$$
r_{0}=l_{B} \sqrt{\frac{2 \gamma E}{\hbar \omega_{0}}}=\sqrt{\frac{2 E}{m \omega_{0}^{2}}},
$$

what exactly corresponds to the classical potential energy $E=V\left(r_{0}\right)=m \omega_{0}^{2} r_{0}^{2} / 2$ of the rotating electron drifting in the confining potential along the circle with the radius $r_{0}$. The single difference of quasi-classical electron behavior from the classical one is that now according to Eq. (34) it moves not along the thin trajectory, but it is spread over the ring with the thickness of order $l_{B}$.

\section{TWO ELECTRONS IN A DOT}

The other good for us example is the two electrons in a parabolic dot because there is the exact solution which can be compared with our approximate results (see, for instance, [9]). In this case the behavior of electrons is described by the following potential:

$$
V\left(\boldsymbol{r}_{1}, \boldsymbol{r}_{2}\right)=\frac{m \omega_{0}^{2}}{2}\left\{r_{1}^{2}+r_{2}^{2}\right\}+\frac{e^{2}}{\left|\boldsymbol{r}_{1}-\boldsymbol{r}_{2}\right|} .
$$


Let us introduce the center of mass and relative motion coordinates. We shall do it in a non standard way in order not to spoil the commutations rules for the fast and slow variables which we already used. Namely, we use the following definition:

$$
\boldsymbol{r}_{\mathrm{c}}=\frac{1}{\sqrt{2}}\left(\boldsymbol{r}_{1}+\boldsymbol{r}_{2}\right), \quad \boldsymbol{r}_{\mathrm{r}}=\frac{1}{\sqrt{2}}\left(\boldsymbol{r}_{1}-\boldsymbol{r}_{2}\right) .
$$

It leads to the separation of variables as the potential can be presented as a sum of two terms

$$
V\left(\boldsymbol{r}_{1}, \boldsymbol{r}_{2}\right)=V_{\mathrm{c}}\left(\boldsymbol{r}_{\mathrm{c}}\right)+V_{\mathrm{r}}\left(\boldsymbol{r}_{\mathrm{r}}\right) .
$$

The potential for the center of mass motion

$$
V_{\mathrm{c}}\left(\boldsymbol{r}_{\mathrm{c}}\right)=\frac{m \omega_{0}^{2}}{2} r_{\mathrm{c}}^{2}
$$

exactly coincides with the single electron potential (27) which was already considered in the previous Section. Consequently, the eigenvalue and eigenfunction of the center of mass motion coincide with those given by Eqs. (29,30). Note that now the capitals $X$ and $Y$ have to be replaced by the slow center of mass motion coordinates $X_{\mathrm{c}}$ and $Y_{\mathrm{c}}$. Performing the same procedure as in the previous Section we shall arrive at center of mass motion density given by Eq. (34) with the coordinate $\boldsymbol{r}$ replaced by the center of mass coordinate $\boldsymbol{r}_{\mathrm{c}}$.

The relative motion potential is given as follows:

$$
V_{\mathrm{r}}\left(\boldsymbol{r}_{\mathrm{r}}\right)=\frac{m \omega_{0}^{2}}{2} r_{\mathrm{r}}^{2}+\frac{e^{2}}{r_{\mathrm{r}} \sqrt{2}} .
$$

According to Eq. (21) it leads to the following slow relative motion Hamiltonian:

$$
H_{\mathrm{r}}=\frac{m \omega_{0}^{2}}{2}\left(R_{\mathrm{r}}^{2}+\frac{l_{B}^{2}}{2}\right)+\frac{e^{2}}{R_{\mathrm{r}} \sqrt{2}}\left(1+\frac{l_{B}^{2}}{4 R_{\mathrm{r}}^{2}}\right) .
$$

The symbol $R_{\mathrm{r}}^{2}$ of course has to be replaced by the operator

$$
R_{\mathrm{r}}^{2} \rightarrow-l_{B}^{4} \frac{\partial^{2}}{\partial X_{\mathrm{r}}^{2}}+X_{\mathrm{r}}^{2}
$$

Consistently the slow motion Schrödinger equation with Hamiltonian (42) can be solved by means of Fourier transformation technique presented in Appendix A. But in this simple case of two electrons one can find the eigenvalues of the above slow motion Hamiltonian rather easily paying attention to the fact that the eigenfunctions of $R_{\mathrm{r}}^{2}$ operator diagonalize Hamiltonian (42) as well. We know the eigenvalues and eigenfunctions of operator $R_{\mathrm{r}}^{2}$ already. They are given by Eqs. (29, 30). Thus, in order to obtain the eigenvalues of Hamiltonian (42) we have to make the following replacement just in the above Hamiltonian:

$$
R_{\mathrm{r}}^{2} \rightarrow l_{B}^{2}(2 n+1) .
$$

Consequently, the relative slow motion eigenvalue reads

$$
E_{n}^{(r)}=\hbar \omega_{0}\left\{\frac{n+1}{\gamma}+\frac{\lambda \sqrt{\gamma}}{\sqrt{2(2 n+1)}}\left[1+\frac{1}{4(2 n+1)}\right]\right\}
$$

where dimensionless parameter of electron-electron interaction $\lambda=l_{0} / a_{B}$ is the ratio of the characteristic confining potential length $l_{0}=\sqrt{\hbar / m \omega_{0}}$ and the Bohr radius $a_{B}=\hbar^{2} / m e^{2}$.

Now adding together eigenvalue (29) for center of mass motion, the relative motion eigenvalue (45), and one more term $\hbar \omega_{c}$ for lowest Landau level energy we obtain the final result for the two electron eigenvalue in the parabolic dot in the slow motion approximation

$$
\begin{aligned}
E_{N, n}= & \hbar \omega_{0}\left\{\gamma+\frac{N+n+2}{\gamma}\right. \\
& \left.+\frac{\lambda \sqrt{\gamma}}{\sqrt{2(2 n+1)}}\left[1+\frac{1}{4(2 n+1)}\right]\right\} .
\end{aligned}
$$

The dimensionless eigenvalue (in $\hbar \omega_{0}$ units) dependencies on the relative magnetic field strengths (on parameter $\left.\gamma=\omega_{c} / \omega_{0}\right)$ for the case of $N=0$ and several $n$ values are shown in Fig. 2a. In Fig. 2b these eigenvalues are compared with the exact solution taken from Merkt paper [9]. We see that when $n \rightarrow \infty$ and $\gamma \rightarrow \infty$ (namely, in the asymptotic region) the approximate consideration is in good agreement with the exact one. Moreover, the quasiclassical treatment describes correctly the main features of the electron behavior in strong magnetic fields, namely, the increment of the angular momentum (the quantum number $n$ plays its role) with the increment of the magnetic field strength. Indeed, minimizing relative motion eigenvalue (45) in respect the magnetic field $\gamma$ in the case of large quantum numbers $n$ we obtain the ground state orbital momentum

$$
n_{0}=(\lambda / 4)^{2 / 3} \gamma
$$

proportional to the magnetic field what agrees with the quantum mechanical result obtained in [10].

Let us also look at the electron density given by the quasi-classical approximation. First, we notice that according what was said above the eigenfunction of Hamiltonian (42) coincides with the eigenfunction of the operator $R_{\mathrm{r}}^{2}$. Thus, it coincides with wave function (30) with the coordinate $X$ replaced by the relative motion coordinate $X_{\mathrm{r}}$. So, performing the same transformation as it was done in Section VII we obtain the relative motion density given by expression (34).

Now taking into account that $N=0$ corresponds to the ground two electon state we can write down the two electron distribution function in the following form:

$$
\begin{aligned}
\rho_{n}\left(\boldsymbol{r}_{1}, \boldsymbol{r}_{2}\right) & \sim \mathrm{e}^{-r_{\mathrm{c}}^{2} / 2 l_{B}^{2}} \cdot\left(r_{\mathrm{r}} / l_{B}\right)^{2 n} \mathrm{e}^{-r_{\mathrm{r}}^{2} / 2 l_{B}^{2}} \\
& \sim\left(\boldsymbol{r}_{1}-\boldsymbol{r}_{2}\right)^{2 n} \mathrm{e}^{-\left(r_{1}^{2}+r_{2}^{2}\right) / 2 l_{B}^{2}} .
\end{aligned}
$$




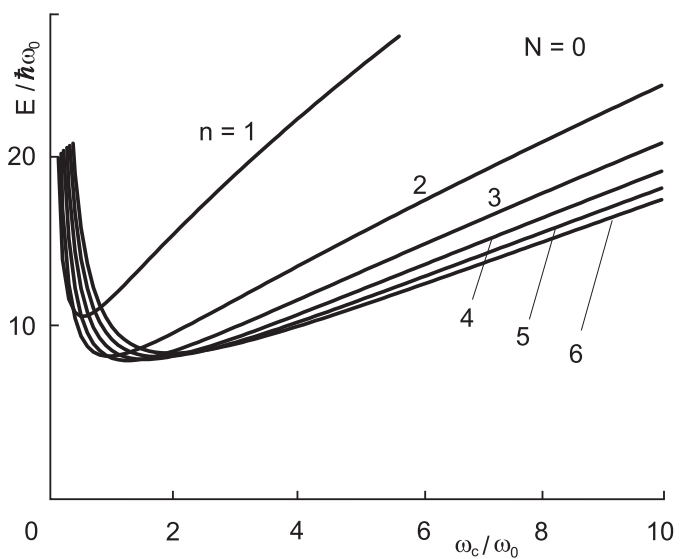

(a)

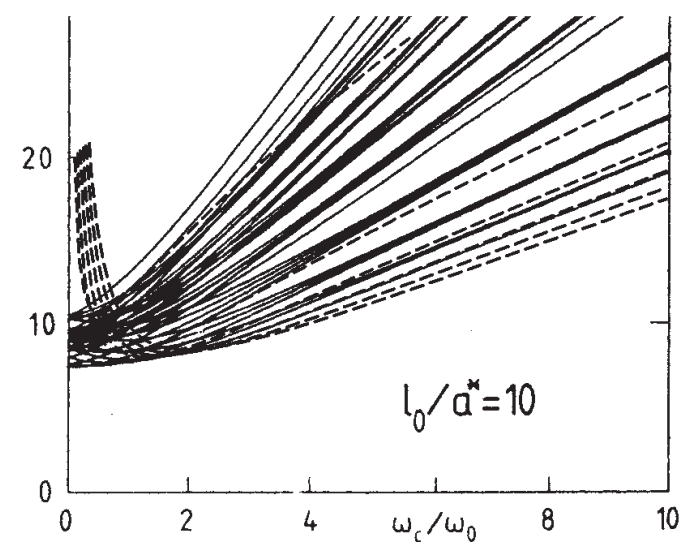

(b)

FIG. 2: Spectrum of two electron system in a parabolic dot: $\mathrm{a}$ - slow motion approximation results; $\mathrm{b}$ - the comparison of approximate results (dashed curves) with the exact results (solid curves) taken from [9].

In Fig. 3 the above function is plotted as a function of the first electron coordinate with the other electron coordinate fixed at the point corresponding to its classical equilibrium position. This point is indicated by solid dot. Actually the plot represents so called pair correlation function. The plot in Fig. 3a corresponds to $n=5$ and the plot in Fig. 3b - to $n=2$. We see that for larger $n$ the pair correlation function demonstrates the peak in the opposite to the fixed electron position what corresponds to the Wigner crystallization of this simple two electron system in the strong magnetic field.

When the magnetic field strength decreases (what corresponds to the ground state with the smaller angular momentum value, say, $n=2$ as it is shown in Fig. $3 \mathrm{~b}$ ) the Wigner crystal starts to melt - the pair correlation function transforms itself from the peak into ring. Note, it demonstrates the fact that the angular melting

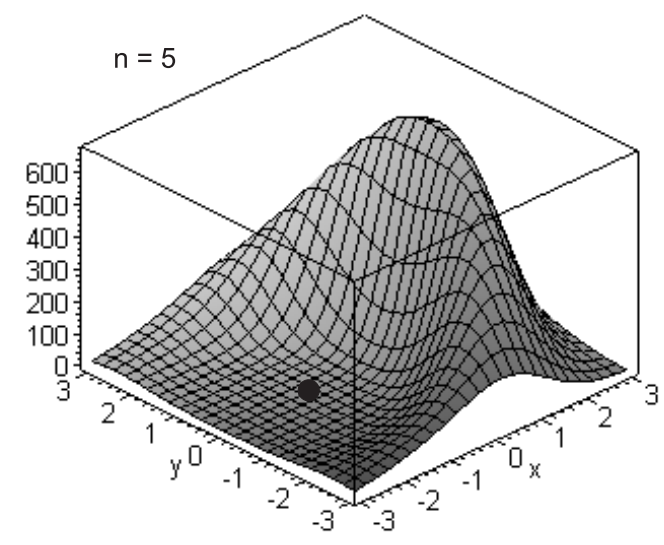

(a)

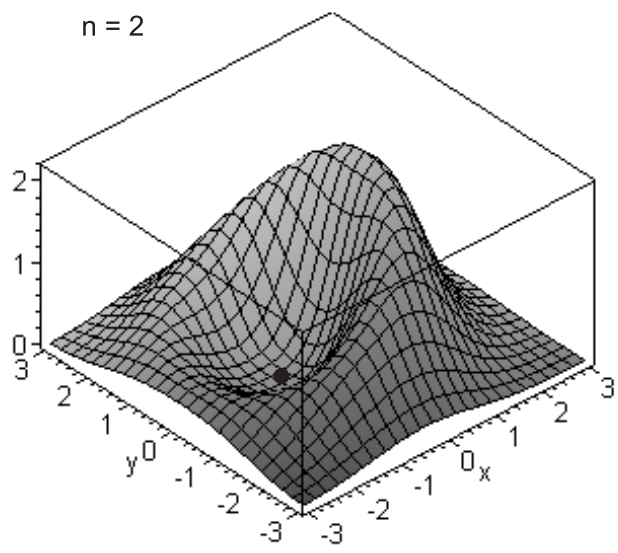

(b)

FIG. 3: Pair-correlation function for various orbital momenta.

precedes the radial one what agrees with quantum mechanical result in [11] obtained for the case without the magnetic field.

\section{CONCLUSIONS}

Let us summarize shortly what was said about the behavior of electrons in the case of the strong magnetic field.

In the asymptotic region of the strong magnetic fields, when all electrons occupy the lowest Landau level only, their kinetic energy is frozen out, and their behavior is guided by weakly varying (characterized by some characteristic length $l_{0}$ ) additional potential. Applying special fast and slow motion variables, the adiabatic procedure and the expansion in $l_{B} / l_{0}$ powers some simplified approximate description can be developed.

In zero order approximation one get the classical equations which describe the electrons as a system of gyro- 
scopes. Those equations actually are the equations for the Larmor circle drift in the gradient of applied potential.

Applying the expansion up to $\left(l_{B} / l_{0}\right)^{2}$ order one obtain the self consistent equation set which coincides with the Schrödinger equation where the role of canonical variables play two cartesian slow motion 2D electron coordinates $X$ and $Y$ with the commutator proportional to the magnetic length squared (instead of being proportional to the Plank constant as it is in the standard Schrödinger equation).

Two simple examples of a single and two electron in a parabolic dot demonstrate the accuracy and main features of proposed approximate description.

The approximate eigenvalues coincide with the exact ones in the asymptotic region when $\gamma=\omega_{c} / \omega_{0} \rightarrow \infty$, and even in the intermediate region $(\gamma \gtrsim 2)$ one can expect rather good semi-quantitative description.

The electron wave functions can be obtained as product of the fast wave function part corresponding to the lowest Landau level and slow wave function part obtained by the above specific slow motion Schrödinger equation. After the transformation to the original variables the wave function obtained in this way describes correctly the quantum mechanical $l_{B} / l_{0}$ order correction to the classical electron motion.

The two electron in a dot example shows that the proposed approximate consideration describes such collective phenomena as the Wigner crystallization, the change of the angular momentum in the ground state when the magnetic field strength increases, the phenomena of angular and radial melting of the Wigner crystal. We hope that this approximate method can be useful for the consideration of more sophisticated many electron systems when the straightforward solution of quantum mechanical equation meets computational difficulties.

\section{Acknowledgments}

I would like to acknowledge Prof. François Peeters and Dr. Bart Partoens from the Antwerp University. Most my ideas on quantum dots appeared during my numerous visits there, due to the close collaboration and the discussions with them. I would like to thank Dr. Egidijus Anisimovas for drawing my attention to various representations of electron wave functions in magnetic field.

\section{APPENDIX A: SLOW MOTION HAMILTONIAN}

As it was mentioned in Section $\mathrm{V}$ performing the adiabatic procedure steps we have to pay attention to the fact that the variables $X$ and $Y$ do not commute each with other. That is why instead of using the straightforward expansion (13) we apply the following Fourier transformation:

$$
\begin{aligned}
V(x, y)= & \frac{1}{2} \int_{-\infty}^{\infty} \frac{d k}{2 \pi} \int_{-\infty}^{\infty} \frac{d q}{2 \pi} \\
& \cdot\left\{\mathrm{e}^{i k x} \mathrm{e}^{i q y}+\mathrm{e}^{i q y} \mathrm{e}^{i k x}\right\} V(k, q), \\
V(k, q)= & \int_{-\infty}^{\infty} d x \int_{-\infty}^{\infty} d y \mathrm{e}^{-i k x} \mathrm{e}^{-i q y} V(x, y) .
\end{aligned}
$$

These two expressions can be considered as a definition of the operator function $V(x, y)$. Thus in the first expression the symbols $x$ and $y$ will be considered as the operators, while in the second one $x$ and $y$ are just the dummy integration variables. The main advantage of such potential representation is that the operators $x$ and $y$ are moved from the general potential function $V(\boldsymbol{r})$ to more simple exponent functions. Although the old $x$ and $y$ variables commute we used the symmetric exponent product which will be necessary in further derivation.

Now we substitute variables (11) into exponents and expand them into $\xi, \eta$-powers:

$$
\begin{aligned}
\mathrm{e}^{i k\left(X+l_{B} \eta\right)} \mathrm{e}^{i q\left(Y-l_{B} \xi\right)} & =\mathrm{e}^{i k X} \mathrm{e}^{i q Y} \mathrm{e}^{i l_{B} k \eta} \mathrm{e}^{-i l_{B} q \xi} \\
& =\mathrm{e}^{i k X} \mathrm{e}^{i q Y}\left\{1+i l_{B} k \eta-\frac{1}{2} l_{B}^{2} k^{2} \eta^{2}\right\}\left\{1-i l_{B} q \xi-\frac{1}{2} l_{B}^{2} q^{2} \xi^{2}\right\} \\
& =\mathrm{e}^{i k X} \mathrm{e}^{i q Y}\left\{1+i l_{B}(k \eta-q \xi)-\frac{1}{2} l_{B}^{2} k^{2} \eta^{2}-\frac{1}{2} l_{B}^{2} q^{2} \xi^{2}+l_{B}^{2} k q \eta \xi\right\}, \\
\mathrm{e}^{i q\left(Y-l_{B} \xi\right)} \mathrm{e}^{i k\left(X+l_{B} \eta\right)} & =\mathrm{e}^{i q Y} \mathrm{e}^{i k X} \mathrm{e}^{-i l_{B} q \xi} \mathrm{e}^{i l_{B} k \eta} \\
& =\mathrm{e}^{i q Y} \mathrm{e}^{i k X}\left\{1+i l_{B}(k \eta-q \xi)-\frac{1}{2} l_{B}^{2} k^{2} \eta^{2}-\frac{1}{2} l_{B}^{2} q^{2} \xi^{2}+l_{B}^{2} k q \xi \eta\right\} .
\end{aligned}
$$

Taking the evident equality

$$
a \eta \xi+b \xi \eta=\frac{1}{2} a(\eta \xi+\xi \eta+i)+\frac{1}{2} b(\xi \eta+\eta \xi-i)=\frac{1}{2}(a+b)(\xi \eta+\eta \xi)+\frac{i}{2}(a-b)
$$


into account we write down the following expansion of the symmetric product of exponents:

$$
\begin{aligned}
& \mathrm{e}^{i k\left(X+l_{B} \eta\right)} \mathrm{e}^{i q\left(Y-l_{B} \xi\right)}+\mathrm{e}^{i q\left(Y-l_{B} \xi\right)} \mathrm{e}^{i k\left(X-l_{B} \eta\right)}=\left\{\mathrm{e}^{i k X} \mathrm{e}^{i q Y}+\mathrm{e}^{i q Y} \mathrm{e}^{i k X}\right\} \\
& \times\left\{1+i l_{B}(k \eta-q \xi)-\frac{1}{2} l_{B}^{2}(k \eta-q \xi)^{2}\right\}+\frac{i}{2} l_{B}^{2} k q\left\{\mathrm{e}^{i k X} \mathrm{e}^{i q Y}-\mathrm{e}^{i q Y} \mathrm{e}^{i k X}\right\} \\
& =2\left[\mathrm{e}^{i k X} \mathrm{e}^{i q Y}\right]^{(S)} L(\xi, \eta, k, q)+i l_{B}^{2} k q\left[\mathrm{e}^{i k X} \mathrm{e}^{i q Y}\right]^{(A)} .
\end{aligned}
$$

Note how the symmetric and antisymmetric exponent products and function $L(\xi, \eta, k, q)$ are defined.

Inserting the above expansion into Fourier transformation (A1), changing the parameters $k$ and $q$ by the operators $i \partial / \partial x$ and $i \partial / \partial y$ acting on exponents, and performing the integration by parts we arrive at the following potential expansion:

$$
\begin{gathered}
V\left(X+l_{B} \eta, Y-l_{B} \xi\right)=\int_{-\infty}^{\infty} d x \int_{-\infty}^{\infty} d y \int_{-\infty}^{\infty} \frac{d k}{2 \pi} \int_{-\infty}^{\infty} \frac{d q}{2 \pi} V(x, y) \\
\times\left\{L(\xi, \eta, k, q)\left[\mathrm{e}^{i k(X-x)} \mathrm{e}^{i q(Y-y)}\right]^{(S)}+\frac{i}{2} l_{B}^{2} k q\left[\mathrm{e}^{i k(X-x)} \mathrm{e}^{i q(Y-y)}\right]^{(A)}\right\} \\
=\int_{-\infty}^{\infty} d x \int_{-\infty}^{\infty} d y \int_{-\infty}^{\infty} \frac{d k}{2 \pi} \int_{-\infty}^{\infty} \frac{d q}{2 \pi} V(x, y) \times\left\{L(\xi, \eta, i \partial / \partial x, i \partial / \partial y)\left[\mathrm{e}^{i k(X-x)} \mathrm{e}^{i q(Y-y)}\right]^{(S)}\right. \\
\left.\quad-\frac{i l_{B}^{2}}{2} \frac{\partial^{2}}{\partial x \partial y}\left[\mathrm{e}^{i k(X-x)} \mathrm{e}^{i q(Y-y)}\right]^{(A)}\right\} \\
=\int d x \int d y \int \frac{d k}{2 \pi} \int \frac{d q}{2 \pi}\left\{\left[\mathrm{e}^{i k(X-x)} \mathrm{e}^{i q(Y-y)}\right]^{(S)} L(\xi, \eta,-i \partial / \partial x,-i \partial / \partial y)\right. \\
\left.\quad-\frac{i l_{B}^{2}}{2}\left[\mathrm{e}^{i k(X-x)} \mathrm{e}^{i q(Y-y)}\right]^{(A)} \frac{\partial^{2}}{\partial x \partial y}\right\} V(x, y) \\
=\int d x \int d y \int \frac{d k}{2 \pi} \int \frac{d q}{2 \pi}\left\{\left[\mathrm{e}^{i k(X-x)} \mathrm{e}^{i q(Y-y)}\right]^{(S)}\left\{1+l_{B}\left(\eta \frac{\partial}{\partial x}-\xi \frac{\partial}{\partial y}\right)-\frac{l_{B}^{2}}{2}\left(\eta \frac{\partial}{\partial x}-\xi \frac{\partial}{\partial y}\right)^{2}\right\}\right. \\
\left.\quad-\frac{i l_{B}^{2}}{2}\left[\mathrm{e}^{i k(X-x)} \mathrm{e}^{i q(Y-y)}\right]^{(A)} \frac{\partial^{2}}{\partial x \partial y}\right\} V(x, y) .
\end{gathered}
$$

Actually it is the definition of the operator function expansion which we have to use instead of expression (13). It can be rewritten in more simple way if we use the following operator function definition:

$$
F^{(X Y)}(X, Y)=\int d x \int d y \int \frac{d k}{2 \pi} \int \frac{d q}{2 \pi} \mathrm{e}^{i k(X-x)} \mathrm{e}^{i q(Y-y)} F(x, y) .
$$

It defines the function with ordered operators - all operators $X$ stand on the left side of the operators $Y$ in all terms of its Taylor expansion, or Fourier transform.

Defining the symmetric and antisymmetric operator function as

$$
\begin{aligned}
& F^{(S)}(X, Y)=\frac{1}{2}\left\{F^{(X Y)}(X, Y)+F^{(Y X)}(X, Y)\right\} \\
& F^{(A)}(X, Y)=\frac{1}{2}\left\{F^{(X Y)}(X, Y)-F^{(Y X)}(X, Y)\right\}
\end{aligned}
$$

we rewrite the potential expansion in the following formal simple form:

$$
\begin{aligned}
V & =\left\{1+l_{B}\left(\eta \frac{\partial}{\partial X}-\xi \frac{\partial}{\partial Y}\right)\right. \\
& \left.-\frac{l_{B}^{2}}{2}\left(\eta \frac{\partial}{\partial X}-\xi \frac{\partial}{\partial Y}\right)^{2}\right\} V^{(S)}+\frac{i l_{B}^{2}}{2} V_{X Y}^{(A)} .
\end{aligned}
$$

Now we are ready to perform the next step of our adiabatic procedure, namely, to insert the obtained potential expansion into fast Hamiltonian (15) and solve the fast eigenvalue problem (18). It can be easily performed using the standard perturbation technique. 
Indeed, using the modified fast Hamiltonian

$$
\begin{aligned}
H_{f} & =H_{0}+H_{1}+H_{2} \\
H_{0} & =\frac{\hbar \omega_{c}}{2}\left(\xi^{2}+\eta^{2}\right) \\
H_{1} & =l_{B}\left(\eta \frac{\partial}{\partial X}-\xi \frac{\partial}{\partial Y}\right) V^{(S)}, \\
H_{2} & =-\frac{l_{B}^{2}}{2}\left(\eta \frac{\partial}{\partial X}-\xi \frac{\partial}{\partial Y}\right)^{2} V^{(S)}
\end{aligned}
$$

we obtain the zero order eigenvalue and function

$$
E_{0}=\frac{\hbar \omega_{c}}{2}, \quad \psi_{0}(\eta)=\pi^{-1 / 4} \mathrm{e}^{-\eta^{2} / 2} .
$$

Next, due to the zero order function symmetry we get $E_{1}=0$, and solving the first order equation

$$
\left\{H_{0}-E_{0}\right\}=-H_{1} \psi_{0}=-l_{B}\left(V_{X}^{(S)}-i V_{Y}^{(S)}\right) \eta \psi_{0}
$$

we define the first order correction to the wave function

$$
\psi_{1}(\eta \mid X, Y)=-\frac{l_{B}\left(V_{X}^{(S)}-i V_{B}^{(S)}\right)}{\hbar \omega_{c}} \eta \psi_{0} .
$$

Then from the second order equation one can easily get the following second order eigenvalue correction:

$$
\begin{gathered}
E_{2}(X, Y)=\int_{-\infty}^{\infty} d \eta \psi_{0}(\eta) H_{2} \psi_{0}(\eta) \\
+\int_{-\infty}^{\infty} d \eta \psi_{0}(\eta) H_{1} \psi_{1}(\eta \mid X, Y) \\
=\frac{l_{B}^{2}}{4}\left\{V_{X X}^{(S)}+V_{Y Y}^{(S)}\right\}-\frac{l_{B}^{2}}{2 \hbar \omega_{c}}\left\{V_{X}^{(S) 2}+V_{Y}^{(S) 2}\right\}
\end{gathered}
$$

Now having the fast motion problem eigenvalue calculated we can proceed with the adiabatic procedure. For this purpose we present the total wave function as

$$
\Psi(\eta, X, t)=\mathrm{e}^{-i E_{0} t}\left\{\psi_{0}(\eta)+\psi_{1}(\eta \mid X, Y)\right\} \Phi(X, t),
$$

insert it into Eq. (11), and obtain the following expression:

$$
\begin{aligned}
& \left\{i \frac{\partial}{\partial t}+E_{0}-H_{f}-V^{(S)}-\frac{i l_{B}^{2}}{2} V_{X Y}^{(A)}\right\} \\
& \quad \cdot\left\{\psi_{0}(\eta)+\psi_{1}(\eta \mid X, Y)\right\} \Phi(X, t)=0
\end{aligned}
$$

Now multiplying the above equation by function $\psi_{0}(\eta)$ from the left side, integrating it over all $-\infty<\eta<\infty$ interval and taking into account the action of the fast Hamiltonian $H_{f}$ on the fast wave function part we arrive at the slow motion equation (19) with the effective Hamiltonian

$$
H=V^{(S)}+\frac{i l_{B}^{2}}{2} V_{X Y}^{(A)}+\frac{l_{B}^{2}}{4} \nabla^{2} V^{(S)}-\frac{l_{B}^{2}}{2 \hbar \omega_{c}}\left\{\nabla V^{(S)}\right\}^{2} .
$$

The performed procedure is consistent at least with the accuracy up to $l_{B}^{2}$. That is why we shall omit the second and the last terms in $(\mathrm{A} 22)$ as they are of order $l_{B}^{4}$. In the last term this dependence appears due to the additional factor $\omega_{c}$ in the denominator, while in the second term it is caused by slow variable commutator (10). Omitting these terms we arrive at the final slow motion Hamiltonian (21).

\section{APPENDIX B: COORDINATE TRANSFORMATION}

According to the ideas of the quantum mechanics we can change the wave function variables by means of the following transformation:

$$
\Psi(x, y)=\int_{-\infty}^{\infty} d \eta \int_{-\infty}^{\infty} d X\langle x, y \mid \eta, X\rangle \Psi(\eta, X)
$$

where the transformation function $\langle x, y \mid \eta, X\rangle$ has to be chosen as the eigenfunction of operators $\hat{x}$ and $\hat{y}$ with the corresponding eigenvalues $x$ and $y$. Namely, this transformation function has to obey the following equations:

$$
\begin{aligned}
& \{\hat{x}-x\}\langle x, y \mid \eta, X\rangle=0 \\
& \{\hat{y}-y\}\langle x, y \mid \eta, X\rangle=0
\end{aligned}
$$

or

$$
\begin{aligned}
\left\{X+l_{B} \eta-x\right\}\langle x, y \mid \eta, X\rangle & =0 \\
\left\{-i l_{B}^{2} \frac{\partial}{\partial X}+i l_{B} \frac{\partial}{\partial \eta}-y\right\}\langle x, y \mid \eta, X\rangle & =0 .
\end{aligned}
$$

It can be checked straightforwardly that the following transformation function satisfies both equations:

$$
\langle x, y \mid \eta, X\rangle=\frac{1}{2 \sqrt{\pi l_{B}}} \mathrm{e}^{i y\left(X-l_{B} \eta\right) / 2 l_{B}^{2}} \delta\left(X+l_{B} \eta-x\right) .
$$

The normalization factor is chosen in agreement with the condition

$$
\begin{aligned}
\int_{-\infty}^{\infty} & d x \int_{-\infty}^{\infty} d y\langle x, y \mid \eta, X\rangle\left\langle x, y \mid \eta^{\prime}, X^{\prime}\right\rangle \\
& =\delta\left(\eta-\eta^{\prime}\right) \delta\left(X-X^{\prime}\right) .
\end{aligned}
$$


[1] L. Jacak, P. Hawrylak, and A. Wójs, Quantum dots (Springer-Verlag, Berlin, 1998).

[2] C. de C. Chamon and X. G. Wen, Phys. Rev. B 49, 8227 (1994).

[3] E. Goldmann and S. R. Renn, cond-mat/9909071 (1999).

[4] K. Hirose and N. S.Wingreen, Phys. Rev. B 59, 4604 (1999).

[5] P. A. Maksym, Phys. Rev. B 53, 10871 (1996).

[6] S. M. Riemann, et all, Phys. Rev. Lett. 83, 3270 (1999).

[7] V. M. Bedanov and F. M. Peeters, Phys. Rev. B 49, 2667 (1994).
[8] I. S. Gradshteyn and I. M. Ryzhik, Table of Integrals, Series and Products (Academic Press, New York, 1994), chap. 7.3 , p. 843.

[9] U. Merkt, J. Huser and M. Wagner, Phys. Rev. B, 43, 7320 (1991).

[10] A. Matulis, F. M. Peeters, Solid St. Comm., 117, 655 (2001).

[11] A. V. Filinov, M. Bonitz, and Yu. E. Lozovik, Phys. Rev. Lett., 86, 3851 (2001). 